\title{
Prophylactic treatment of osteoporosis after SCl: promising research, but not yet indicated
}

\author{
Dustin Anderson ${ }^{1} \cdot$ Andrew J Park ${ }^{1}{ }^{1}$
}

Received: 27 November 2018 / Accepted: 13 December 2018

(๑) International Spinal Cord Society 2019

\begin{abstract}
In persons with spinal cord injury (SCI), osteoporosis and associated fragility fractures are a prevalent phenomenon with clinically meaningful morbidity and mortality. Prevention of osteoporosis utilizing both physical modalities and pharmacological therapies is an area of high-clinical importance. In our perspective, the current body of research cannot provide clear guidance on prophylactic interventions to prevent osteoporosis specifically to stratify SCI subjects to their risk for fragility fractures. Without this critical research, clinicians cannot weigh the risk versus benefits of interventions, such as bisphosphonates, which is not a benign treatment. Other treatments such as physical modalities provide little risk and have other therapeutic benefit. This perspective is an argument that the current research does not indicate prophylactic pharmacological intervention to prevent osteoporosis in the SCI population.
\end{abstract}

\section{Introduction}

Bone mass is known to rapidly deteriorate during the acute stage of spinal cord injury (SCI) and continues to decline over several decades [1]. Fracture incidence after SCI is $\sim 1 \%$ per year and increases to $3.4-4.6 \% 10$ years post injury, which leads to a cumulative lifetime fracture rate of $>40 \%$ [1]. Fragility fractures can lead to significant medical complications and impact quality of life. They are associated with prolonged hospitalizations with risk for respiratory illness, urinary tract infections, delirium, amputation, and death [2]. Moreover, they can lead to chronic complications including contracture formation, increased spasticity, and pain [3]. Fragility fractures in SCI have several clinically important differences in comparison to the general population. Most notably, clinically relevant fracture sites in SCI are in the distal femur and proximal tibia, distinct from non-injured populations, which includes hip, radius, and lumbar spine [2]. Given the prevalence of osteoporosis and associated fragility fractures in SCI, it is important for clinicians to consider prevention options to

Andrew J Park

andrew.park@ucdenver.edu

1 Department of Physical Medicine and Rehabilitation, University of Colorado School of Medicine, Aurora, CO, USA mitigate clinically meaningful morbidity and mortality. Several prophylactic interventions during the acute stage of SCI has been evaluated with various physical methods and pharmacologic therapies with varying efficacy.

Osteoporosis is diagnosed in SCI based on bone density similar to the general population. For post-menopausal women and men age 50 or older, T-score can be used to classify bone density according to the World Health Organization (WHO) definitions of normal (T-score $>-1$ ), osteopenia (T-score $<-1$ and $>-2.5$ ) and osteoporosis (Tscore $<-2.5)$. For premenopausal women and men under the age of 50, Z-score can be used to classify bone density at the hip as normal $(Z$-score $>-2)$ or as lower than expected for age and sex $(Z$-score $<-2)$. In cases when osteoporosis is diagnosed by DXA, there are published paradigms supporting pharmacological treatment [4]. This provides a good rationale for early screening during the acute phase of SCI. This perspective is an argument that the current body of research cannot recommend prophylactic pharmacological intervention to prevent osteoporosis after SCI.

Several classes of medications are used to treat osteoporosis in both the general population and after SCI. Bisphosphonates (BP) are among the most commonly used anti-resorptive medications. Systematic reviews and metaanalyses have reviewed BP therapies to prevent osteoporosis in acute SCI [5, 6], and we defer full review of the literature to these publications. Overall, BP therapy after acute SCI may attenuate bone mineral density (BMD) loss 
in comparison to no treatment if administered acutely after injury. Bryson et al. notes that definition of acuity, BP administered, length of treatment, subject demographics including level and completeness of injury were heterogenous and difficult to interpret in some cases. Although some degree of BMD attenuation was observed in majority of the studies, the author did not recommend prophylactic treatment with BP noting methodological and evidence level concerns [6]. In a subsequent prospective trial, Zoledronic acid (ZA) was administered in open label to 13 patients with acute SCI within 3 months of injury, 6 treated with $\mathrm{ZA}$ and 7 receiving no treatment. The treatment group demonstrated attenuation of BMD compared to the control group in the hip at 6 and 12 months consistent with previous trials. Contrary to the findings at the hip, however, the treatment group had a greater loss in BMD compared to the control group at the distal femur and proximal tibia at 6 and 12 months. The authors concluded that it would not be prudent to recommend the use of ZA due to the lack of efficacy at the knee region, the most common site for chronic SCI fracture [7]. Other medications, such as the recombinant parathyroid hormone teriparatide and monoclonal antibody anti-RANKL denosumab have additionally been studied in the SCI population with promising results compared to control [8, 9]. Currently, FDA guidelines do not suggest prophylactic use of bisphosphonates to prevent osteoporosis development in the general population and suggest drug holidays in active treatment of osteoporosis as prolonged treatment has been associated with rare, but serious complications relating to over-suppression of bone metabolism [10]. These include osteonecrosis of the jaw and increased non-healing acetabular femur fractures [11]. Similar guidelines do not exist in SCI and to our knowledge has not been studied.

Some interventions such as physical modalities provide a relatively low risk compared to possible benefits. Mechanical loading is the most profound stimulus for bone remodeling, and many studies have investigated various methods and frequencies of interventions to increase or reduce loss of BMD. Body weight supported locomotor training demonstrated reversal of muscle atrophy, however no change in BMD after 6 months of training [12]. Functional electrical stimulation (FES) has also been studied as a promising modality either cycling or rowing, which has shown improvement initially in BMD. However, sustaining BMD requires ongoing FES training with rapid bone loss observed after ceasing FES [13-17]. Additionally, a metaanalysis of FES cycling observed that programs with frequency $>5$ days per week is required to produce reduction in bone loss in comparison to controls, while $\leq 3$ days per week did not demonstrate significant BMD changes [5]. There is no doubt that mechanical loading is a key component in the prevention of osteoporosis, but the current research cannot conclude on a specific program and intervention that is feasible in the clinical setting. Nonetheless, considering the well documented benefits of exercise and relatively low risk of side effects from the intervention, physical modalities is surely encouraged in all populations. Further research into clinically meaningful mechanical loading program is necessary.

\section{Conclusion}

Osteoporosis and fragility fractures are prevalent and clinically meaningful in the SCI population. Prevention is a critical area of research. Although many osteoporosis medications demonstrate variable degrees of ameliorating BMD loss, SCI clinicians currently lack the guidance on length of pharmacological therapy necessary to weigh the risks and benefits of medication administration. Physical modalities have added benefits related to cardiovascular health and should always be encouraged, but variability in intervention frequency and intensity have demonstrated inconclusive results. Because of these reasons, we argue that the current research does not indicate for prophylactic treatment to prevent osteoporosis in the SCI population.

\section{Compliance with ethical standards}

Conflict of interest Dustin Anderson and Andrew J Park declare that they have no conflict of interest.

Publisher's note: Springer Nature remains neutral with regard to jurisdictional claims in published maps and institutional affiliations.

\section{References}

1. Zehnder Y, Lüthi M, Michel D, Knecht H, Perrelet R, Neto I, et al. Long-term changes in bone metabolism, bone mineral density, quantitative ultrasound parameters, and fracture incidence after spinal cord injury: a cross-sectional observational study in 100 paraplegic men. Osteoporos Int. 2004;15:180-9.

2. Troy KL, Morse LR. Measurement of bone: diagnosis of SCIinduced osteoporosis and fracture risk prediction. Top Spinal Cord Inj Rehabil. 2015;21:267-74.

3. Bauman WA, Cardozo CP. Osteoporosis in individuals with spinal cord injury. PM R. 2015;7:188-201. quiz 201.

4. Zleik N, Weaver F, Harmon RL, Le B, Radhakrishnan R, JirauRosaly WD, et al. Prevention and management of osteoporosis and osteoporotic fractures in persons with a spinal cord injury or disorder: a systematic scoping review. J Spinal Cord Med. 2018;1-25.

5. Chang K-V, Hung C-Y, Chen W-S, Lai M-S, Chien K-L, Han D$\mathrm{S}$. Effectiveness of bisphosphonate analogues and functional electrical stimulation on attenuating post-injury osteoporosis in spinal cord injury patients- a systematic review and meta-analysis. PLoS ONE. 2013;8:e81124. 
6. Bryson JE, Gourlay ML. Bisphosphonate use in acute and chronic spinal cord injury: a systematic review. J Spinal Cord Med. 2009;32:215-25.

7. Bauman WA, Cirnigliaro CM, La Fountaine MF, Martinez L, Kirshblum SC, Spungen AM. Zoledronic acid administration failed to prevent bone loss at the knee in persons with acute spinal cord injury: an observational cohort study. J Bone Miner Metab. 2015;33:410-21.

8. Gordon KE, Wald MJ, Schnitzer TJ. Effect of parathyroid hormone combined with gait training on bone density and bone architecture in people with chronic spinal cord injury. PM R. 2013;5:663-71.

9. Gifre L, Vidal J, Carrasco JL, Muxi A, Portell E, Monegal A, et al. Denosumab increases sublesional bone mass in osteoporotic individuals with recent spinal cord injury. Osteoporos Int. 2016;27:405-10.

10. Managing Osteoporosis in Patients on Long-Term Bisphosphonate Treatment: Report of a Task Force of the American Society for Bone and Mineral Research. - PubMed - NCBI [Internet]. [cited 2018 Sep 11]. https://www-ncbi-nlm-nih-gov. proxy.hsl.ucdenver.edu/pubmed/26350171

11. Whitaker M, Guo J, Kehoe T, Benson G. Bisphosphonates for osteoporosis-where do we go from here? N Engl J Med. 2012;366:2048-51.
12. Giangregorio LM, Hicks AL, Webber CE, Phillips SM, Craven BC, Bugaresti JM, et al. Body weight supported treadmill training in acute spinal cord injury: impact on muscle and bone. Spinal Cord. 2005;43:649-57.

13. Lai C-H, Chang WH-S, Chan WP, Peng C-W, Shen L-K, Chen J$\mathrm{JJ}$, et al. Effects of functional electrical stimulation cycling exercise on bone mineral density loss in the early stages of spinal cord injury. J Rehabil Med. 2010;42:150-4.

14. Eser P, de Bruin ED, Telley I, Lechner HE, Knecht H, Stüssi E. Effect of electrical stimulation-induced cycling on bone mineral density in spinal cord-injured patients. Eur $\mathrm{J}$ Clin Invest. 2003;33:412-9.

15. Bélanger M, Stein RB, Wheeler GD, Gordon T, Leduc B. Electrical stimulation: can it increase muscle strength and reverse osteopenia in spinal cord injured individuals? Arch Phys Med Rehabil. 2000;81:1090-8.

16. Chen S-C, Lai C-H, Chan WP, Huang M-H, Tsai H-W, Chen J-JJ. Increases in bone mineral density after functional electrical stimulation cycling exercises in spinal cord injured patients. Disabil Rehabil. 2005;27:1337-41.

17. Mohr T, Podenphant J, Biering-Sorensen F, Galbo H, Thamsborg $\mathrm{G}$, Kjaer M. Increased bone mineral density after prolonged electrically induced cycle training of paralyzed limbs in spinal cord injured man. Calcif Tissue Int. 1997;61:22-5. 